Supporting Information

\title{
Light-driven nanodroplet generation using porous membranes
}

Rui Feng, $\neq$ Qixiang Wang, $\neq$ Yiming Qiao, Runheng Yang, Shun An, Fanchen Meng, Shengtao Yu,Wei Hao, Benwei Fu, Peng Tao, Kehang Cui, Chengyi Song, * Wen Shang* and Tao Deng*

Center of Hydrogen Science, School of Materials Science and Engineering, Shanghai Jiao Tong University, 800 Dong Chuan Road, Shanghai 200240, P. R. China. 


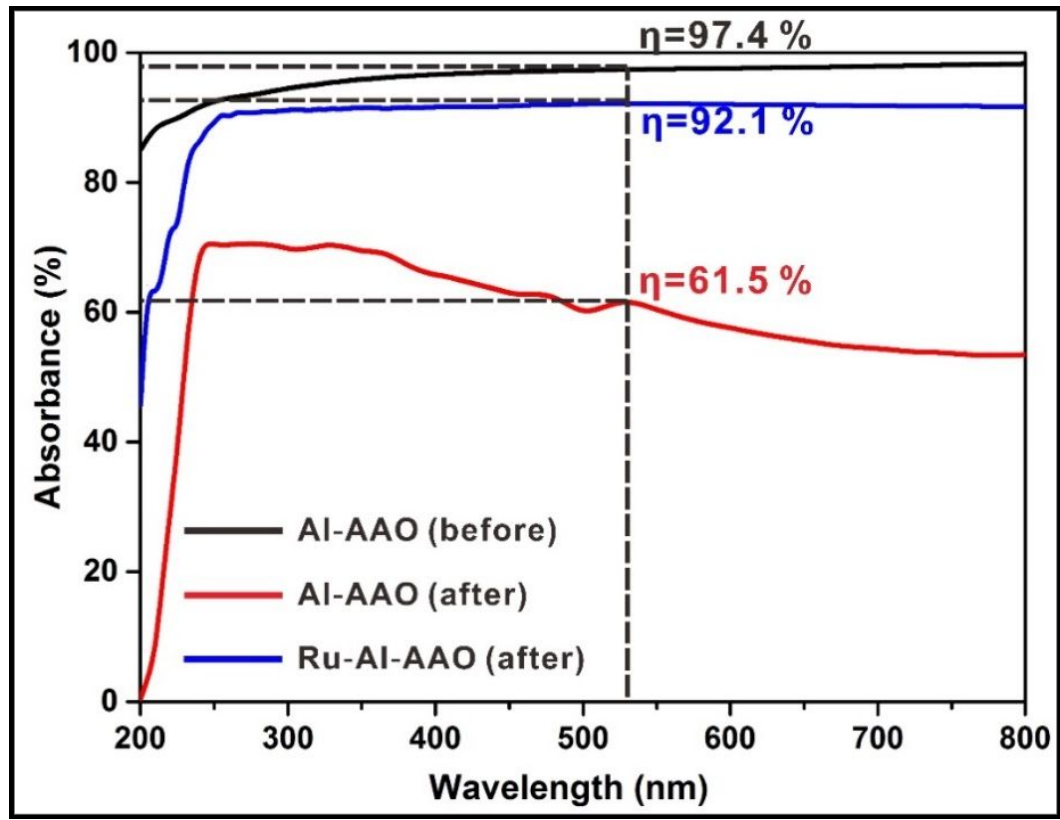

Figure S1. The UV-Vis spectra of the original Al-AAO membrane (black curve), the Al-AAO membrane after laser illumination (red curve), and the Ru-Al-AAO membrane after laser illumination (blue curve). 


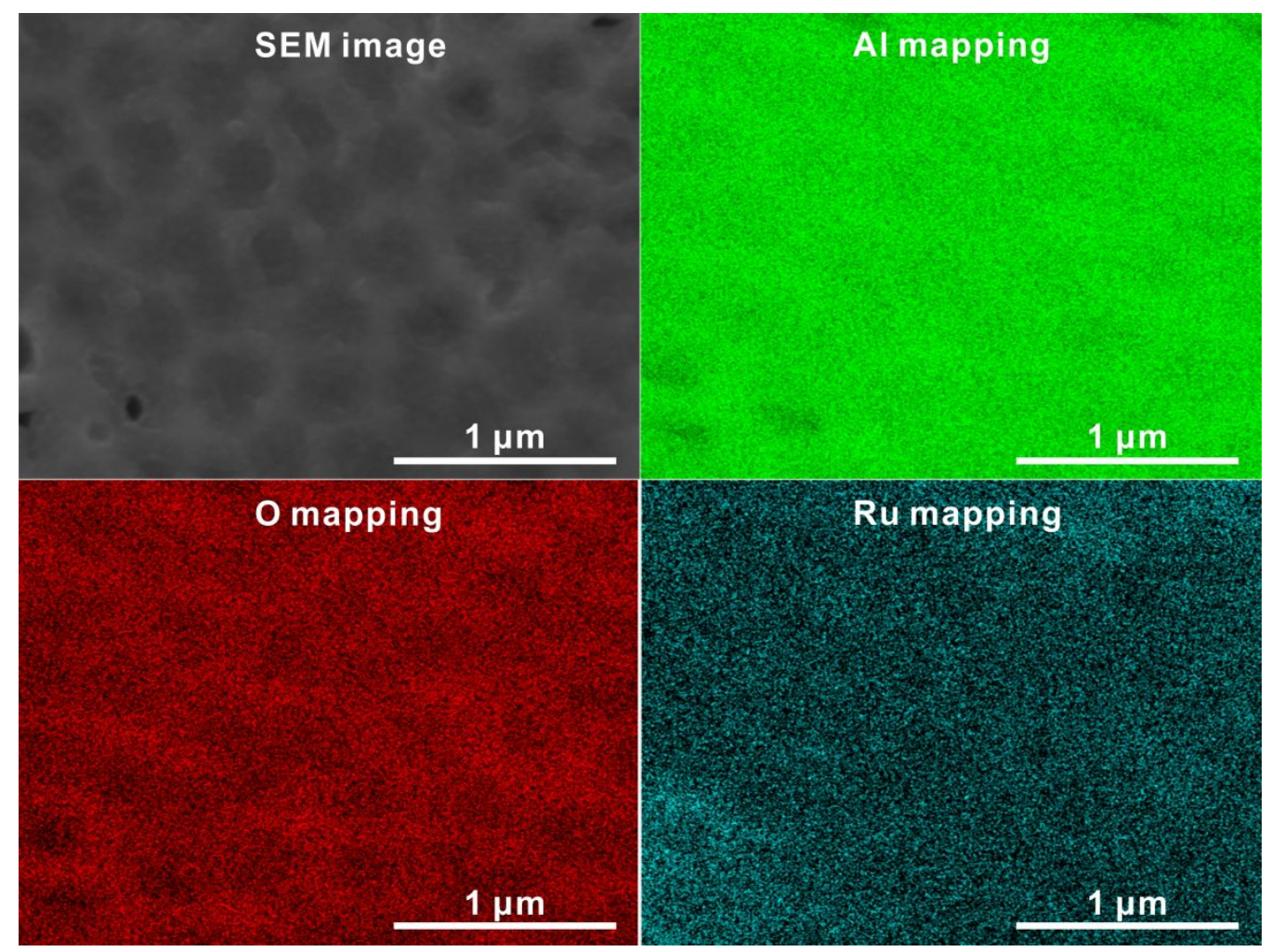

Figure S2. The elemental mapping of the pre-sintered area on the front view of Ru-Al-AAO membrane through the energy dispersive X-ray spectroscopy (EDS). 


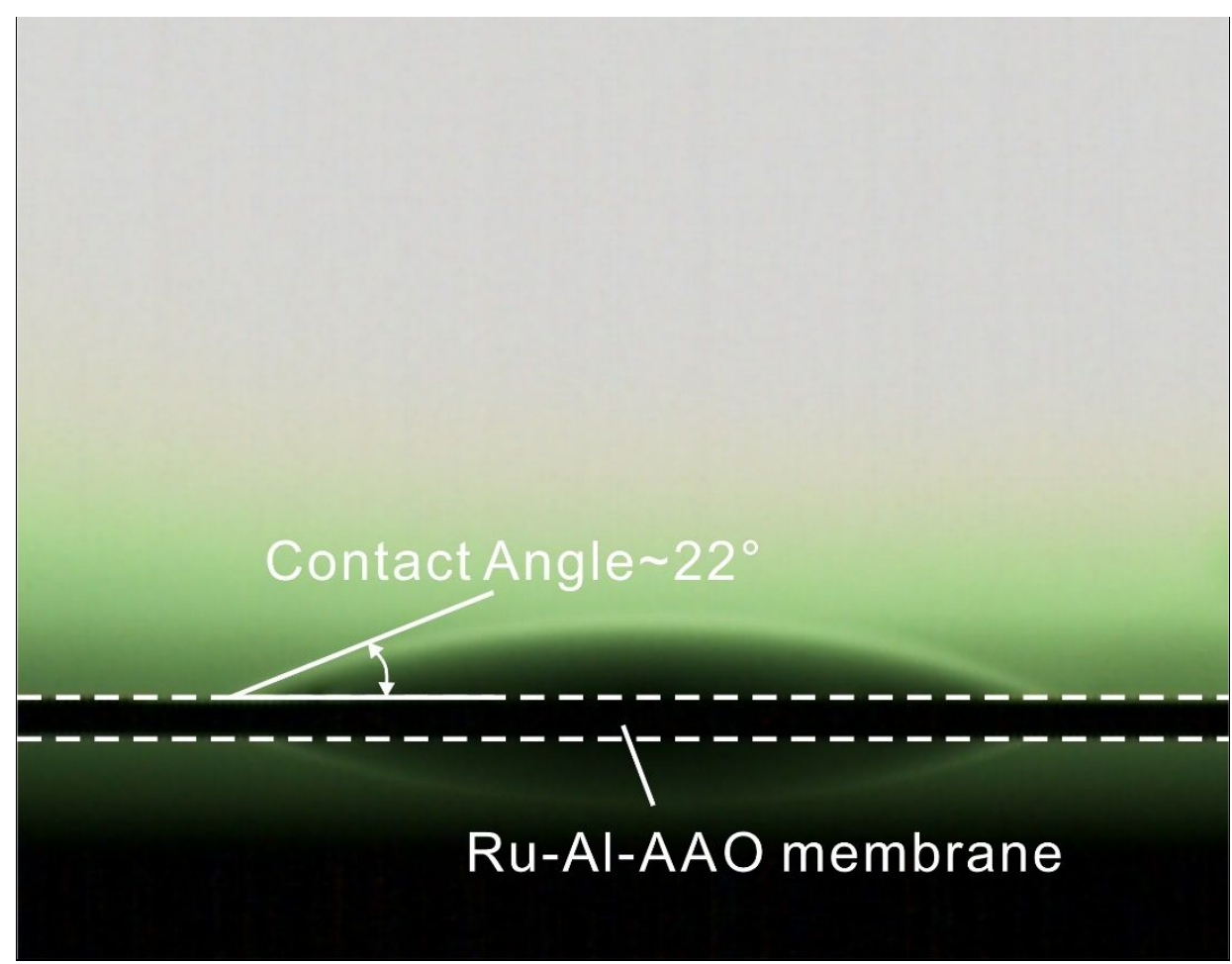

Figure S3. The contact angle of the back-surface of the Ru-Al-AAO membrane. The Ru coating shows hydrophilic nature due to its native oxide layer on top of the membrane. ${ }^{1,2}$ 


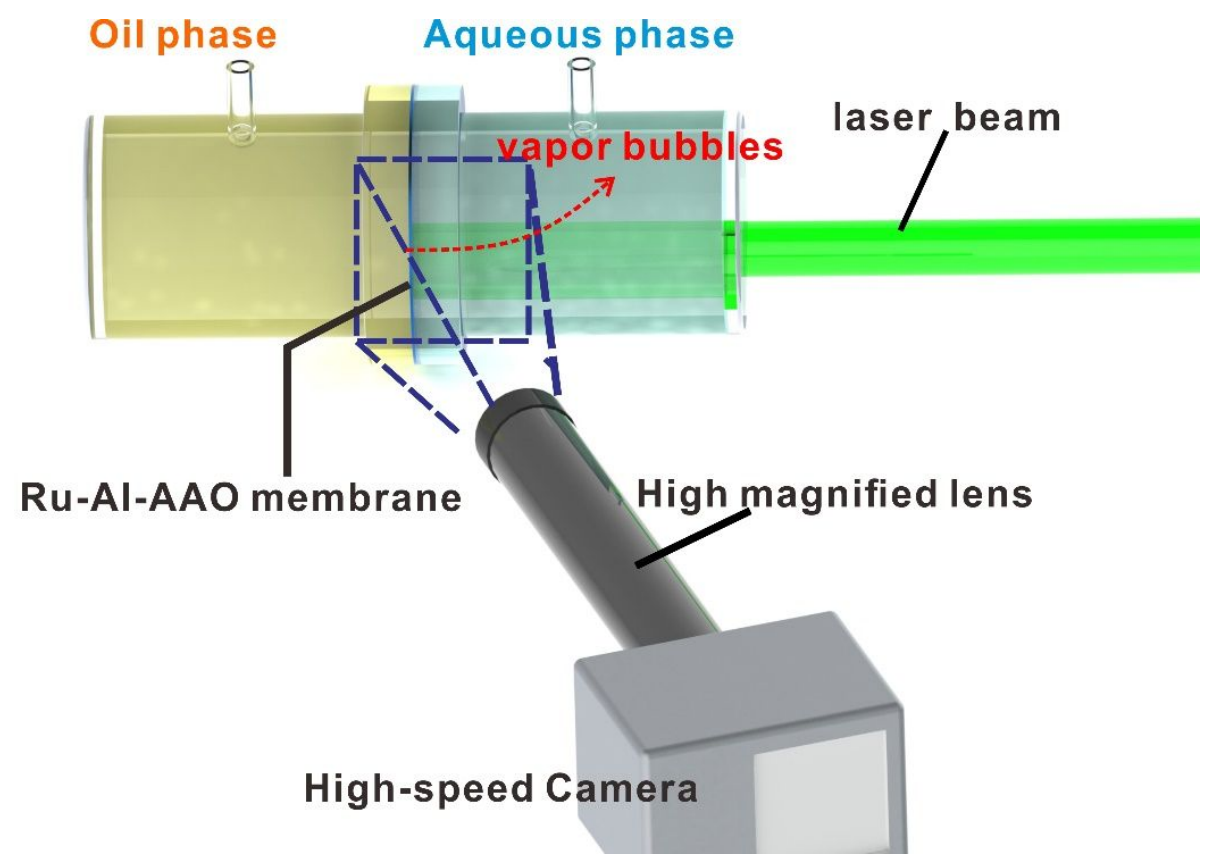

Figure S4. The setup of visualization system. A high speed camera (S-VIT LS, AOS technologies AG, Switzerland) equipped with a high-magnification lens (12X zoom lens and $1 \mathrm{X}$ adapter, Navitar, Inc, USA) was used to capture the vapor bubble release process from the laser illumination area. The high-speed camera was set at an angle of $30^{\circ}$ with respect to the surface of the membranes. The frame rate in this visualization experiment was $200 \mathrm{fps}$. 
Table S1. Influence of surfactant concentration on droplet size (HLB=9.65).

\begin{tabular}{|l|c|c|c|c|}
\hline$c_{\mathrm{CTAB}} / \mathrm{mM}$ & 0.06 & 0.09 & 0.12 & 0.24 \\
\hline Droplet size/nm & 60.5 & 62.5 & 150.6 & 118.9 \\
\hline
\end{tabular}

Table S2. Influence of HLB value on droplet size $\left(c_{\mathrm{CTAB}}=0.09 \mathrm{mM}\right)$.

\begin{tabular}{|l|c|c|c|c|}
\hline HLB value & 9.65 & 11 & 12.5 & 14 \\
\hline Droplet size/nm & 62.5 & 63.6 & 125.6 & 161.0 \\
\hline
\end{tabular}

Table S1 and Table S2 show the results of the experiments with different concentration of surfactants that can stabilize the nanoemulsion. ${ }^{3}$ In Table S1, we controlled the HLB value to be 9.65 as we changed the concentration of $\mathrm{CTAB}$, which shows that droplet size increases as the concentration of CTAB increases. As we fixed the centration of CTAB to $0.09 \mathrm{mM}$, Table $\mathbf{S 2}$ shows that the droplet size also increases with the increase of the HLB value. In the experiment for Table S2, the total weight of Span 80 and Tween 80 was fixed to be $15 \mathrm{wt} \%$ of the oil phase and we changed their relative ratio to control the HLB value of the oil phase. 
Table S3. Comparison of production rate with other's work.

\begin{tabular}{|l|l|}
\hline Approaches & Production Rate \\
\hline This work (light-driven membrane emulsification) & $\sim 9.8 \mathrm{~L} / \mathrm{m}^{2} \cdot \mathrm{h}$ \\
\hline Membrane emulsification, nanoemulsion, nanoparticle, drug delivery ${ }^{4}$ & $4.5-19.2 \mathrm{~L} / \mathrm{m}^{2} \cdot \mathrm{h}$ \\
\hline Membrane emulsification, nanoemulsion, nanoparticle $^{5}$ & $11.36 \mathrm{~L} / \mathrm{m}^{2} \cdot \mathrm{h}$ \\
\hline Membrane emulsification, nanoemulsion $^{6}$ & $0.01 \mathrm{~L} / \mathrm{m}^{2} \cdot \mathrm{h}$ \\
\hline
\end{tabular}

To obtain the production rate of the light-driven emulsification, we estimated the active area based on the image analysis of the videos captured by the high speed camera and also through CFD simulation. The active area is the area where there is active pressure perturbation that leads to the generation of the nanodroplets. The active area includes the bubble generation area and the area surrounding the bubble generation area with efficient pressure disturbance that generates the nanodroplets. The overall active area is estimated to be $\sim 0.5 \mathrm{~mm}$ in diameter and $\sim 0.196 \mathrm{~mm}^{2}$ in surface area. The production rate for the nanodroplets can be calculated by Equation $\mathrm{S}(1)$ :

$$
q=\frac{c \% \times m / \rho_{o}}{S \times t}
$$

Where $\mathrm{c} \%$ is the weight percent of nanodroplets in the water phase measured by TGA, $m$ is the mass of nanoemulsion obtained, $\rho_{o}$ is the density of the oil phase, $S$ is the surface area of the active area, and $t$ is the illumination time. The production rate of one-beam experiment obtained after $2 \mathrm{~h}$ of illumination was thus calculated to be $\sim 9.8 \mathrm{~L} / \mathrm{m}^{2} \bullet \mathrm{h}$. 

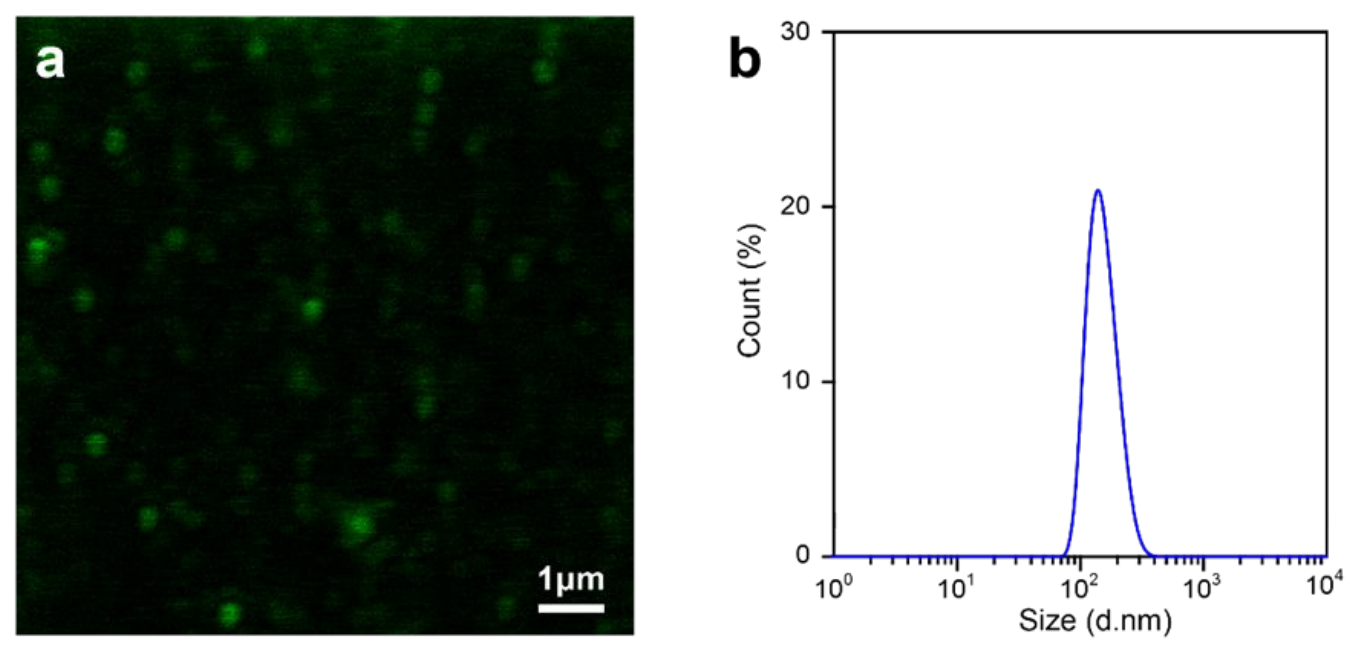

Figure S5. Characterization of the nanoemulsion with the fluorescent dye. (a) Fluorescent image of nanodroplets dispersed in the water phase. (b) Size distribution of nanodroplets with the fluorescent dye.

The nanodroplets with the fluorescent dye was generated by using 3,3'-dioctadecyloxacarbocyanine perchlorate $\left(\operatorname{DiOC}_{18}(3)\right)$, which is a lipophilic fluorescent dye with green light emission. In the experiment, we added $0.5 \mathrm{ml}$ of the fluorescent dye solution (10mg DiOC18(3) dissolved in $1 \mathrm{ml}$ dimethyl sulfoxide (DMSO)) into $9.5 \mathrm{ml}$ of the oil phase $(\mathrm{HLB}=9.56$ and the concentration of CTAB $=0.09 \mathrm{mM})$. After the laser illumination we observed nanodroplets with green fluorescence dispersed in the water phase under the confocal microscope (Leica TCS SP8 STED) (Figure S5 (a)).The nanodroplets have an average size of $260 \mathrm{~nm}$ under the confocal microscope, which is larger than the size measured from the DLS ( $190 \mathrm{~nm}$, Figure S5 (b)). Such size difference might be due to the impact of the dye solution to the interfacial tension between different phases, 

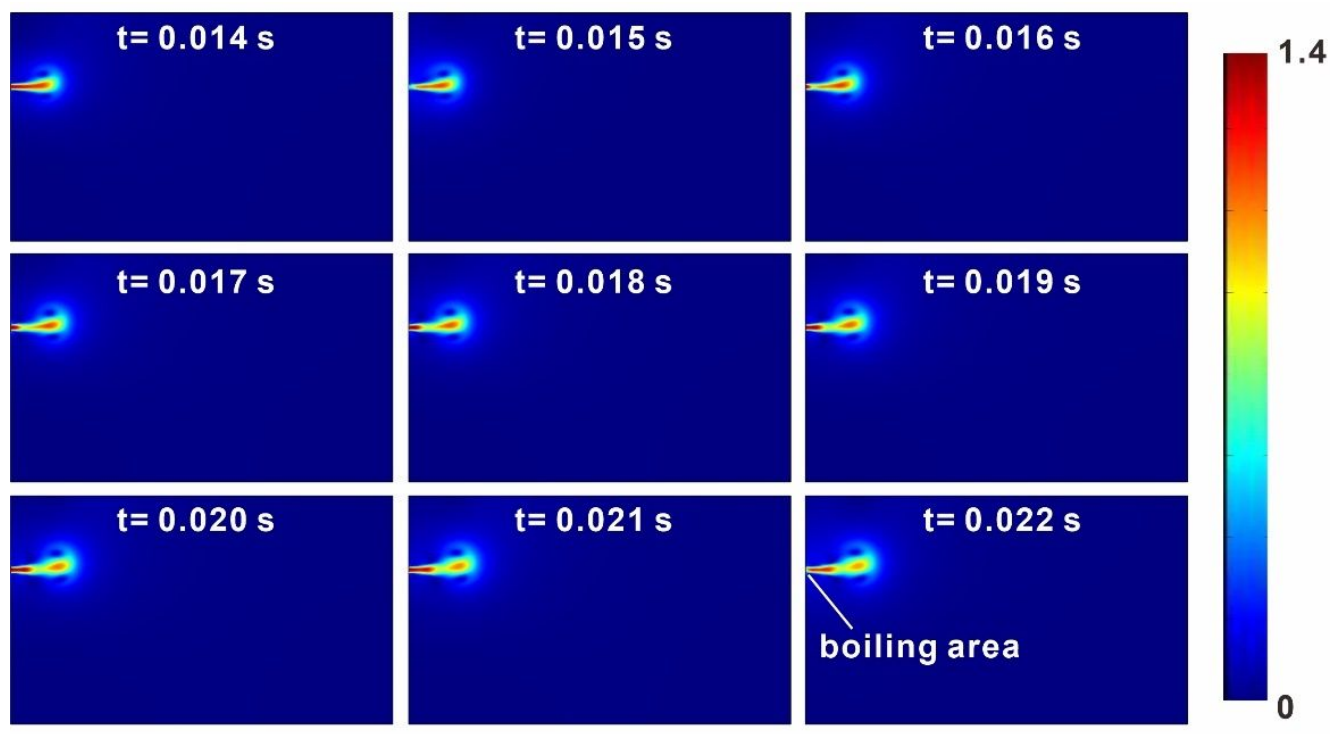

velocity $(\mathrm{m} / \mathrm{s})$
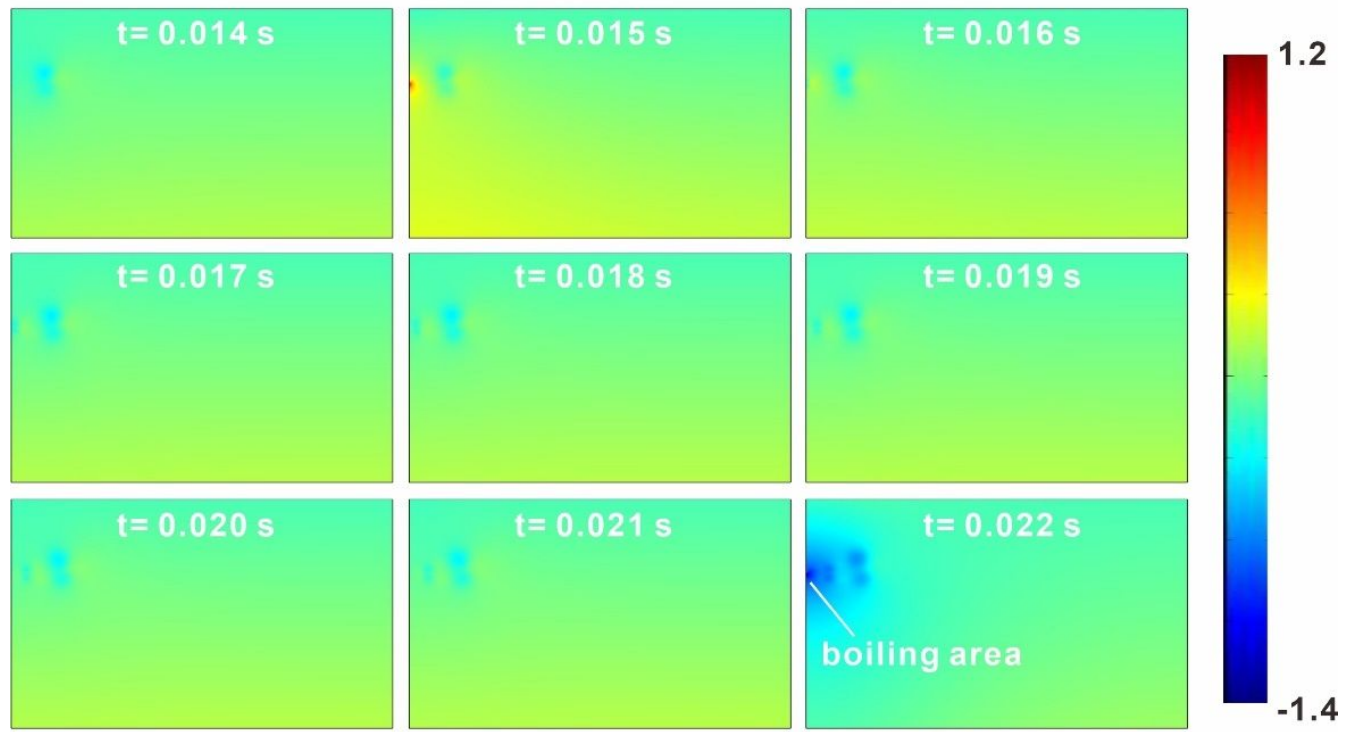

pressure $(\mathrm{kPa})$

Figure S6. The subsequent cycle of releasing a vapor bubble into the aqueous phase $(0.014 \mathrm{~s}$ to $0.022 \mathrm{~s}$ ). (a) The velocity distribution in the aqueous phase during the subsequent cycle. (b) The pressure distribution in the aqueous phase during the subsequent cycle. The local pressure sharply rises to the maximum of $1.2 \mathrm{kPa}$ at $\mathrm{t}=0.015 \mathrm{~s}$ and drops to a negative value with minimum of 1.4 $\mathrm{kPa}$ at $\mathrm{t}=0.022 \mathrm{~s}$. 


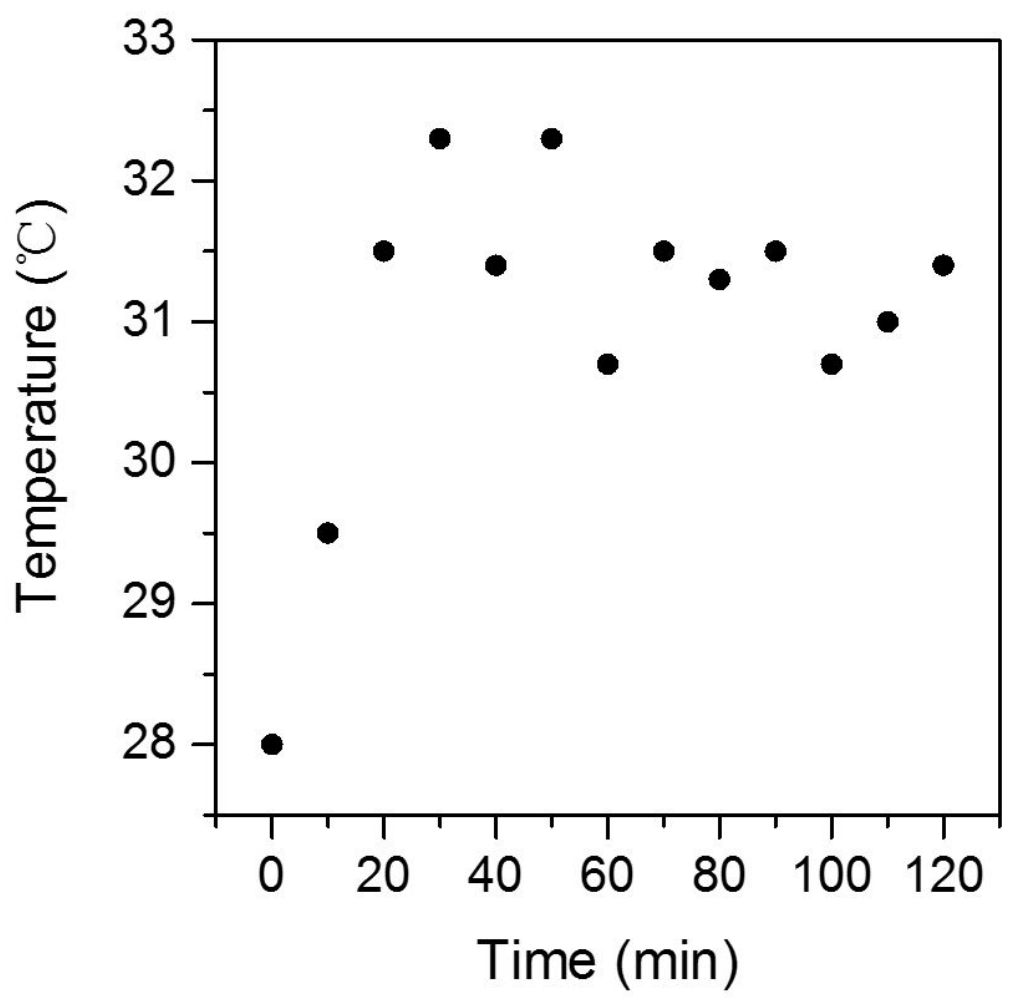

Figure S7. The measured temperature of oil phase during $2 \mathrm{~h}$ of laser illumination. 


\section{REFERENCE}

(1) Shaheen, A.; Sturm, J. M.; Ricciardi, R.; Huskens, J.; Lee, C. J.; Bijkerk, F. Characterization of Self-Assembled Monolayers on a Ruthenium Surface. Langmuir 2017, 33, 6419-6426.

(2) Amanapu, H. P.; Sagi, K. V.; Teugels, L. G.; Babu, S. V. Role of Guanidine Carbonate and Crystal Orientation on Chemical Mechanical Polishing of Ruthenium Films. ECS Journal of Solid State Science and Technology 2013, 2, P445-P451.

(3) Feng, J.; Nunes, J. K.; Shin, S.; Yan, J.; Kong, Y. L.; Prud'homme, R. K.; Arnaudov, L. N.; Stoyanov, S. D.; Stone, H. A. A Scalable Platform for Functional Nanomaterials via Bubble-Bursting. Adv. Mater. 2016, 28, 4047-4052.

(4) Guo, P.; Martin, C. R.; Zhao, Y.; Ge, J.; Zare, R. N. General Method for Producing Organic Nanoparticles Using Nanoporous Membranes. Nano Lett. 2010, 10, 2202-2206.

(5) Medina-Llamas, M.; Mattia, D. Semi-Continuous Production of Iron Oxide Nanoparticles via Membrane Emulsification. Appl. Surf. Sci. 2019, 463, 504-512.

(6) Gehrmann, S.; Bunjes, H. Instrumented Small Scale Extruder to Investigate the Influence of Process Parameters during Premix Membrane Emulsification. Chemical Engineering Journal 2016, 284, 716 723. 